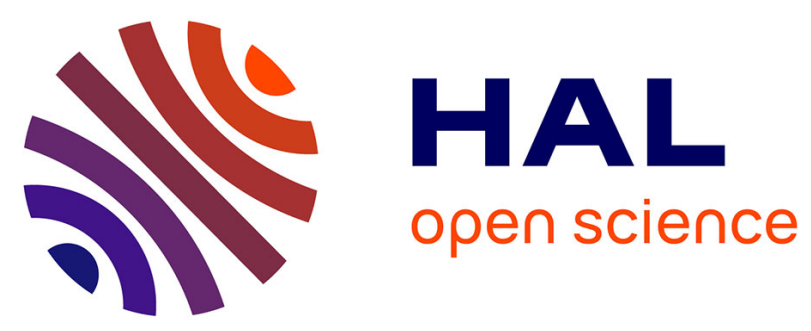

\title{
A Complex Sprectrum Based SAR Image Resampling Method With Restricted Target Sidelobes and Statistics Preservation
}

\author{
Rémy Abergel, Saïd Ladjal, Florence Tupin, Jean-Marie Nicolas
}

\section{- To cite this version:}

Rémy Abergel, Saïd Ladjal, Florence Tupin, Jean-Marie Nicolas. A Complex Sprectrum Based SAR Image Resampling Method With Restricted Target Sidelobes and Statistics Preservation. 2017 IEEE International Geoscience and Remote Sensing Symposium, Jul 2017, Fort Worth, United States. 10.1109/IGARSS.2017.8128214 . hal-01447608

\section{HAL Id: hal-01447608 https://hal-imt.archives-ouvertes.fr/hal-01447608}

Submitted on 27 Jan 2017

HAL is a multi-disciplinary open access archive for the deposit and dissemination of scientific research documents, whether they are published or not. The documents may come from teaching and research institutions in France or abroad, or from public or private research centers.
L'archive ouverte pluridisciplinaire HAL, est destinée au dépôt et à la diffusion de documents scientifiques de niveau recherche, publiés ou non, émanant des établissements d'enseignement et de recherche français ou étrangers, des laboratoires publics ou privés. 


\title{
A COMPLEX SPECTRUM BASED SAR IMAGE RESAMPLING METHOD WITH RESTRICTED TARGET SIDELOBES AND STATISTICS PRESERVATION
}

\author{
Rémy Abergel, Saïd Ladjal, Florence Tupin, and Jean-Marie Nicolas (first.last@telecom-paristech.fr) \\ LTCI, Télécom ParisTech, Université Paris Saclay, Paris, France
}

\begin{abstract}
The aim of this work is to present a resampling scheme for SAR images that preserves spatial resolution and produces statistically accurate images at the same time. Indeed, SAR images are, for reasons due to their acquisition process, well sampled signals according to the Shannon sampling theory. In the presence of strong responses, that we will refer to as targets, a sinc-like function centered at the target is smeared over the entire image and is particularly visible in the range of tens of pixels surrounding the target. To mitigate this phenomenon, the usual solution is to apply an apodization window in the Fourier domain so as to change the cardinal sine impulse response into a much rapidly decaying one. This approach has two major drawbacks. It reduces the resolution of the image and introduces inaccurate statistical dependency between pixels. We propose to resample the image in an adaptive and robust way so that the target smear is canceled and the new sampled image is completely faithful to the underlying signal.
\end{abstract}

Index Terms - Shannon interpolation, complex spectrum, total variation, targets, subpixellic image processing

\section{INTRODUCTION}

SAR images are provided by complex signal processing being at the heart of the SAR technique (range compression, SAR synthesis). The raw data received by the antenna before these operations are usually not provided by space agencies. The provided Single Look Complex data (SLC) are affected by two important factors that can be seen in the complex Fourier spectrum of the image: over-sampling and weighting of the azimuth and range spectrum [1]. These factors can change depending on the data provider even for similar resolutions of the SLC images. For instance, the weighting functions of TerraSAR-X and CSK images are different. These processing have a strong impact on the appearance of the images (spreading of the strong targets) and induce a correlation between neighboring pixels, which can affect further processing like physical parameter estimation [2]. In this paper we investigate how the complex spectrum information can be taken into account to improve SAR images by unweighting images while limiting sidelobes of the impulse response of strong targets, and we show how to produce an image suitable both for visualization and further processing. The paper is organized as follows. Section 2 introduces the notations and gives a method to cancel apodization when the weighting function is unknown. Section 3 presents the proposed resampling scheme and is concluded by a proof of statistical accuracy of the result under a reasonable model.

This work is supported by the ANR through the MIRIAM project.

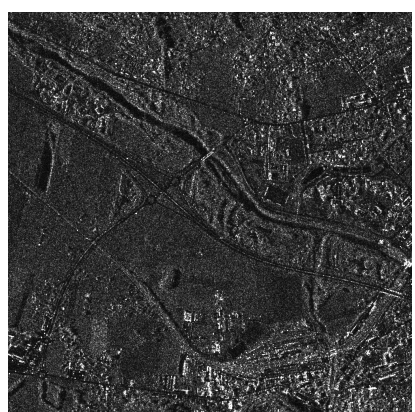

(a) amplitude image

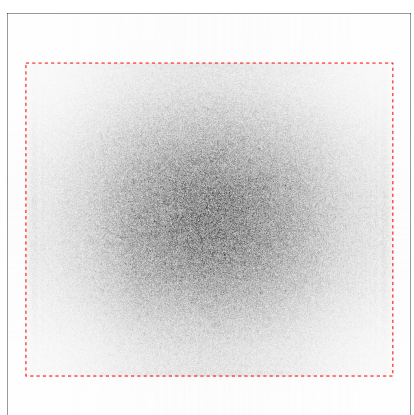

(b) Fourier spectrum
Fig. 1. Example of TerraSAR-X image data. We display in (a) the modulus of a SAR image $u: \Omega \rightarrow \mathbb{C}$, and in (b) the modulus of $\widehat{u}$ over a single period $\widehat{\Omega}$ (high values are displayed in dark, low values in bright, white meaning zero). The Fourier spectrum (b) reveals the presence of zero-padding ( $\widehat{u}$ is zero everywhere outside of a rectangular sub-frequency domain $\widehat{\omega} \subsetneq \widehat{\Omega}$ delimited by the red dashed line), showing that the image $u$ was oversampled.

\section{FROM THE SAR IMAGE DATA TO THE PSEUDO-RAW SPECTRUM}

\subsection{Pseudo-raw image and pseudo-raw spectrum}

Let $u: \Omega \rightarrow \mathbb{C}$ denote a complex-valued SAR image with domain $\Omega=I_{M} \times I_{N}$, where $I_{K}=\{0, \ldots, K-1\}$. We denote by $\widehat{u}$ the discrete Fourier transform (DFT) of $u$, which is the two-dimensional and $(M, N)$-periodic signal defined by

$$
\forall(\alpha, \beta) \in \mathbb{Z}^{2}, \quad \widehat{u}(\alpha, \beta)=\sum_{(k, l) \in \Omega} u(k, l) e^{2 i \pi\left(\frac{\alpha k}{M}+\frac{\beta l}{N}\right)} .
$$

We note $\widehat{I}_{K}=\left[-\frac{K}{2}, \frac{K}{2}\right) \cap \mathbb{Z}$, then we set $\widehat{\Omega}=\widehat{I}_{M} \times \widehat{I}_{N}$, which represents the cannonical frequency domain associated to $\Omega$ and is also a period of $\widehat{u}$.

We display in Fig. 1 the modulus of a SLC TerraSAR-X image $u$ and the modulus of $\widehat{u}$. We see on that particular example that $\widehat{u}$ is non-zero on a rectangular sub-frequency domain $\widehat{\omega}=\widehat{I}_{m} \times \widehat{I}_{n} \subset \widehat{\Omega}$ (for some given integers $m$ and $n$ such as $m \leq M, n \leq N$ ), and zero outside, showing that $u$ was oversampled. Besides, it happens that the non-zero part of the Fourier spectrum is in fact apodized, which means that it resulted from a multiplication in the Fourier domain by a frequency attenuating function. This function results from the weighting affecting the antenna pattern and the weighting applied to the data [1] which depends on the data provider.

In the following, we assume that for any $(\alpha, \beta) \in \widehat{\Omega}$, we have 


$$
\widehat{u}(\alpha, \beta)=\left\{\begin{array}{cl}
\widehat{u_{0}}(\alpha, \beta) \cdot \gamma(\alpha, \beta) & \text { if }(\alpha, \beta) \in \widehat{\omega} \\
0 & \text { otherwise, }
\end{array}\right.
$$

where $\widehat{\omega}=\widehat{I}_{m} \times \widehat{I}_{n} \subset \widehat{\Omega}, \gamma: \widehat{\omega} \rightarrow \mathbb{R}_{++}$is a known frequency attenuating function and $\widehat{u_{0}}: \widehat{\omega} \rightarrow \mathbb{C}$ is called the pseudo-raw spectrum. We call pseudo-raw image the complex-valued image $u_{0}: \omega \rightarrow \mathbb{C}$ with spatial domain $\omega=I_{m} \times I_{n}$ obtained by taking the inverse DFT of $\widehat{u_{0}}$. Since $u_{0}$ and $u$ have different resolutions, the pseudoraw image will be compared to the image $u_{\omega}: \omega \rightarrow \mathbb{C}$ obtained by removing the zero-padding from $\widehat{u}$, that is, the image defined in the Fourier domain by $\widehat{u_{\omega}}(\alpha, \beta)=\widehat{u}(\alpha, \beta)$ for all $(\alpha, \beta) \in \widehat{\omega}$.

\subsection{Practical estimation of the pseudo-raw spectrum}

Now, let us focus on the inversion of (2), that is, on the computation of the pseudo-raw spectrum $\widehat{u_{0}}$. When the subfrequency domain $\widehat{\omega}$ and the frequency attenuating function $\gamma$ are known (for instance provided by the spatial agency who generated the image) the relation (2) can be easily inverted and we get

$$
\forall(\alpha, \beta) \in \widehat{\omega}, \quad \widehat{u_{0}}(\alpha, \beta)=\frac{\widehat{u}(\alpha, \beta)}{\gamma(\alpha, \beta)} .
$$

When those pieces of information are unavailable or lost, as we assume in all the experiments that we propose in this document, they must be estimated. We make the reasonable assumption that the subfrequency domain $\widehat{\omega}$ can be easily retrieved by looking at the Fourier spectrum $\widehat{u}$, as it is the case in Fig. 1 . In the case $\gamma$ is not explicitly known, we propose to consider the following separable estimate given by

$$
\forall(\alpha, \beta) \in \widehat{\omega}, \quad \gamma(\alpha, \beta)=a_{\gamma} \cdot \gamma_{1}(\alpha) \cdot \gamma_{2}(\beta),
$$

where $a_{\gamma} \in \mathbb{R}_{++}$is a normalization factor whose setting is discussed below, and $\gamma_{1}, \gamma_{2}$ are the signals obtained by averaging $\left|\widehat{u_{\omega}}\right|$ in both directions, that is, by setting for all $(\alpha, \beta) \in \widehat{\omega}$,

$$
\gamma_{1}(\alpha)=\frac{1}{n} \sum_{\beta \in \widehat{I}_{n}}\left|\widehat{u_{\omega}}(\alpha, \beta)\right|, \quad \gamma_{2}(\beta)=\frac{1}{m} \sum_{\alpha \in \widehat{I}_{m}}\left|\widehat{u_{\omega}}(\alpha, \beta)\right|,
$$

which yields two positively valued functions $\gamma_{1}: \widehat{I}_{m} \rightarrow \mathbb{R}_{++}$and $\gamma_{2}: \widehat{I}_{n} \rightarrow \mathbb{R}_{++}$as soon as $\left|\widehat{u_{\omega}}\right|$ does not have a column or line being identically-zero. The normalization factor $a_{\gamma}$ is computed in order that $\left|u_{0}\right|$ and $\left|u_{\omega}\right|$ assume the same maximum over $\omega$, which yields

$$
a_{\gamma}=\frac{\max _{(k, l) \in \omega}\left|u_{0}^{\prime}(k, l)\right|}{\max _{(k, l) \in \omega}\left|u_{\omega}(k, l)\right|},
$$

where $u_{0}^{\prime}$ is such that $\widehat{u_{0}^{\prime}}(\alpha, \beta)=\widehat{u_{\omega}}(\alpha, \beta) /\left(\gamma_{1}(\alpha) \cdot \gamma_{2}(\beta)\right)$ for all $(\alpha, \beta) \in \widehat{\omega}$. Finally, $u_{0}$ can by computed using (3), (4) and (5).

In Fig. 2, we display a cropping of the amplitude images $\left|u_{\omega}\right|$ and $\left|u_{0}\right|$ computed from the image $u$ of Fig. 1. As expected the unweighted image is better localized (finer localization of the information) at the price of a large spreading of strong targets in azimuth and range directions.

\section{ADAPTATIVE RESAMPLING SCHEME FOR THE PSEUDO-RAW IMAGE}

\subsection{Model}

We illustrate in Fig. 3 that the bright targets observed on the pseudoraw image can be very well approached by a two-dimensional cardinal sine function defined by (as given by the SAR processing [1]):

$$
\forall(x, y) \in \mathbb{R}^{2}, \quad \operatorname{sinc}(x, y)=\frac{\sin (\pi x)}{\pi x} \cdot \frac{\sin (\pi y)}{\pi y},
$$

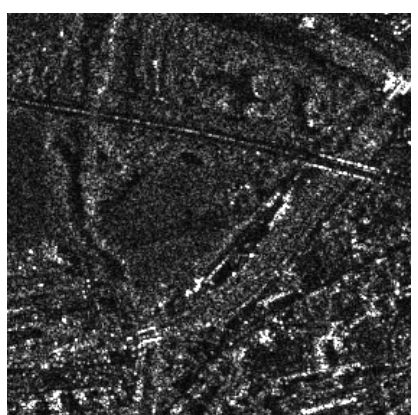

modulus of $u_{\omega}$

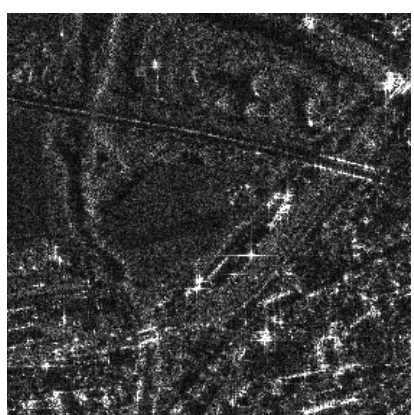

modulus of $u_{0}$ (pseudo-raw)
Fig. 2. Comparison between apodized and pseudo-raw images. Removing the apodization from $u_{\omega}$ yields the pseudo-raw image $u_{0}$, we display here the modulus of a subpart of those two images. We can see that the pseudo-raw image $u_{0}$ shows a better level of details than $u_{\omega}$, as well as a more precise localization of the information. However, in the presence of strong targets, the signal is polluted by horizontal and vertical patterns.

with the continuity-preserving condition $\frac{\sin (0)}{0}=1$. An interpretation of this phenomenon is that the target is sufficiently narrow to be transformed, by the acquisition process, to the impulse response, yielding the cardinal sine function. When the position of the target does not coincide with the sampling grid, an oscillatory pattern peculiar to the sinc function pollutes the values of the pixels in the vicinity of the target, yielding (we drop the double indexes for the spatial coordinates in this section) an observed signal of the type

$$
\forall k \in \omega, \quad u_{0}(k)=A \operatorname{sinc}\left(k-\left(k_{0}+\delta\right)\right)+u_{0}^{*}(k),
$$

where $A \in \mathbb{C}$ denotes the target amplitude, $k_{0}+\delta$ (with $k_{0} \in \omega$, $\delta \in\left[-\frac{1}{2}, \frac{1}{2}\right]^{2}$ ) the subpixellic position of the target, and $u_{0}^{*}$ the signal that would be acquired in the absence of the target.

The obvious solution to this problem is to resample the image on a grid such that the coordinates of the target are integers, thus suppressing the side lobes contributions. In order to do that, one has to estimate the subpixellic position of the target and then perform a translation of the image by the vector $t=-\delta$. Indeed, noting $U_{0}: \mathbb{R}^{2} \rightarrow \mathbb{C}$ the Shannon interpolate of $u_{0}$ which is computed as $U_{0}=U_{0}^{r}+i \cdot U_{0}^{i}$, where $U_{0}^{r}$ and $U_{0}^{i}$ denote the (real-valued) Shannon interpolates of the real and imaginary parts of $u_{0}$ (see for instance [3] for the explicit definition), the translated signal is $v_{0}$ : $k \mapsto U_{0}(k+\delta)$ and satisfies

$$
\forall k \in \omega, \quad v_{0}(k)=U_{0}^{*}(k+\delta)+\left\{\begin{array}{cl}
A & \text { if } k=k_{0} \\
0 & \text { otherwise, }
\end{array}\right.
$$

where $U_{0}^{*}$ denotes the Shannon interpolate of $u_{0}^{*}$. We see that, contrary to $u_{0}$, the resampled signal $v_{0}$ is not polluted anymore by the oscillations of the cardinal sine.

Since in practice, there may and will be numerous targets in a single image, a global translation will not be sufficient to accommodate all the targets of the image. For that reason, we propose to devise a local scheme to compute, at each position in the image, the translation that reduces the interference of the target-induced cardinal sine. Indeed, contrary to $[4,5]$, we made the choice to not explicitly detect targets to keep the process as robust as possible.

\subsection{Local displacement vector field}

Our approach consists in computing from $u_{0}$ a dense field of displacements $T=\left(T_{x}, T_{y}\right): \omega \rightarrow\left[-\frac{1}{2}, \frac{1}{2}\right] \times\left[-\frac{1}{2}, \frac{1}{2}\right]$, and resample 


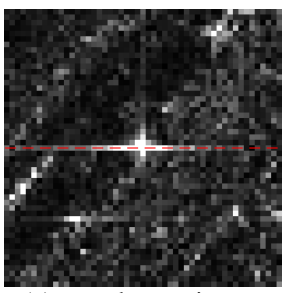

(a) pseudo-raw image

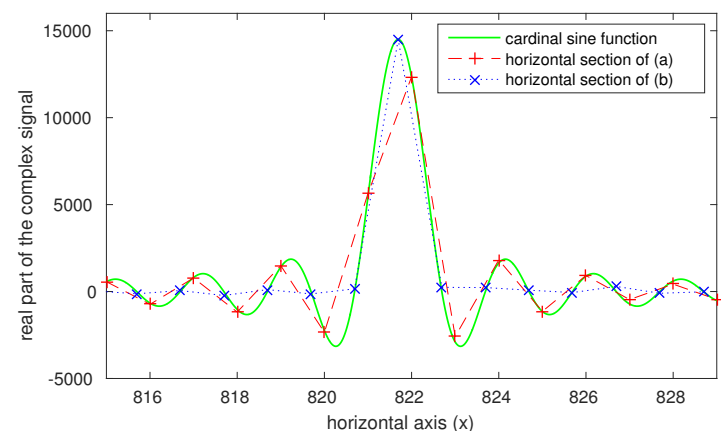

Fig. 3. Resampling targets at the subpixellic scale. We display in (a) the modulus of the pseudo-raw image $u_{0}$ in the vinicity of a strong target. We display in (b) and (c) the modulus of the image $u_{0}$ resampled by means of a translation of vector $t=(0.3,0)$ and $t=(0.3,0.1)$ respectively, yielding more localized signals. We display in the second row a plot of the real part of an horizontal section of $u_{0}$, yielding the red dashed curve, and we do the same for the resampled signal displayed in (b), yielding the blue dotted curve. The green plain curve represents a pure cardinal sine, that is a function of the type $x \mapsto A \operatorname{sinc}(x)$ where $A \in \mathbb{R}$. We see that the samples of both red and blue signals match very well with a sampling of the cardinal sine, the same observation holds for the imaginary parts of the considered signals.

the image $u_{0}$ over the locally translated grid, which means to compute the image $v_{0}: \omega \rightarrow \mathbb{C}$ defined by

$$
\forall(k, l) \in \omega, \quad v_{0}(k, l)=U_{0}\left(k-T_{x}(k, l), l-T_{y}(k, l)\right) .
$$

In practice, the two components $T_{x}$ and $T_{y}$ of $T$ will be estimated independently, we describe here the computation of $T_{x}$, that of $T_{y}$ being totally similar.

Given a locality parameter $K \in \mathbb{N}$ (we took $K=25$ in all our experiments) and a position $\left(k_{0}, l_{0}\right) \in \omega$, we associate to each translation $t_{x} \in\left[-\frac{1}{2}, \frac{1}{2}\right]$ the mono-dimensional signal $v_{t_{x}} \in \mathbb{C}^{2 K+1}$ corresponding to the restriction of the horizontally translated signal $(x, y) \mapsto U_{0}\left(x-t_{x}, y\right)$ to the horizontal $2 K+1$ neighborhood of the pixel $\left(k_{0}, l_{0}\right)$, that is,

$$
\forall p \in[-K, K] \cap \mathbb{Z}, \quad v_{t_{x}}(p)=U_{0}\left(k_{0}-p-t_{x}, l_{0}\right) .
$$

We propose to select among all candidate translations the one that minimizes a particular cost function $t_{x} \mapsto J\left(v_{t_{x}}\right)$ where $J: \mathbb{C}^{2 K+1} \rightarrow \mathbb{R}$, yielding

$$
T_{x}\left(k_{0}, l_{0}\right)=\underset{-\frac{1}{2} \leq t_{x}<\frac{1}{2}}{\operatorname{argmin}} J\left(v_{t_{x}}\right) .
$$

We considered three different choices for $J$, denoted below as $J_{1}$, $J_{2}$ and $J_{3}$. (i) $J_{1}\left(v_{t_{x}}\right)=-\max _{p \in[-K, K]}\left|v_{t_{x}}(p)\right|$. The idea underlying this choice is that when a target exists in the vicinity of $\left(k_{0}, l_{0}\right)$, the appropriate translation is found by looking for the signal $v_{t_{x}}$ having the highest maximal modulus (as it is for instance the case for the blue dotted curve in Fig. 3).

(ii) $J_{2}\left(v_{t_{x}}\right)=\mathrm{TV}^{\mathrm{d}}\left(v_{t_{x}}^{r}\right)+\mathrm{TV}^{\mathrm{d}}\left(v_{t_{x}}^{i}\right)$, noting $v_{t_{x}}^{r}$ and $v_{t_{x}}^{i}$ the real and imaginary parts of $v_{t_{x}}$, and $\mathrm{TV}^{\mathrm{d}}$ the discrete total variation operator defined by

$$
\forall w \in \mathbb{R}^{2 K+1}, \quad \operatorname{TV}^{\mathrm{d}}(w)=\sum_{-K \leq p<K}|w(p+1)-w(p)| .
$$

The idea is that, when sampled on the appropriate grid, the discrete total variation of a target-induced cardinal sine is minimal, whereas it is always higher for all non integer displacements of the grid (the red dashed curve in Fig. 3 is more oscillatory than the blue dotted curve and exhibits a higher discrete total variation).

(iii) $J_{3}\left(v_{t_{x}}\right)=\mathrm{TV}_{\text {mask }}^{\mathrm{d}}\left(v_{t_{x}}^{r}\right)+\mathrm{TV}_{\text {mask }}^{\mathrm{d}}\left(v_{t_{x}}^{i}\right), \mathrm{TV}_{\text {mask }}^{\mathrm{d}}$ being defined for any $w \in \mathbb{R}^{2 K+1}$ by

$$
\mathrm{TV}_{\text {mask }}^{\mathrm{d}}(w)=\sum_{\substack{-K \leq p<K \\ p \notin\left\{p_{0}-1, p_{0}\right\}}}|w(p+1)-w(p)|,
$$

where $p_{0}$ denotes the position where $|w|$ is maximal. Compared to the previous criterion, we choose to mask the contribution of the brightest pixel to the total variation so that the $\mathrm{TV}_{\text {mask }}^{\mathrm{d}}$ of a pure cardinal sine function sampled on the appropriate grid is zero.

Since our numerical expriments revealed that the third choice led to the most satisfying results, it was systematically used in all the experimental results displayed below. The computation of the resampled image $v_{0}$ defined by (9) from the pseudo-raw image $u_{0}$ is summarized in Algorithm 1, and some experimental results are displayed and commented in Fig. 4 and Fig. 5.

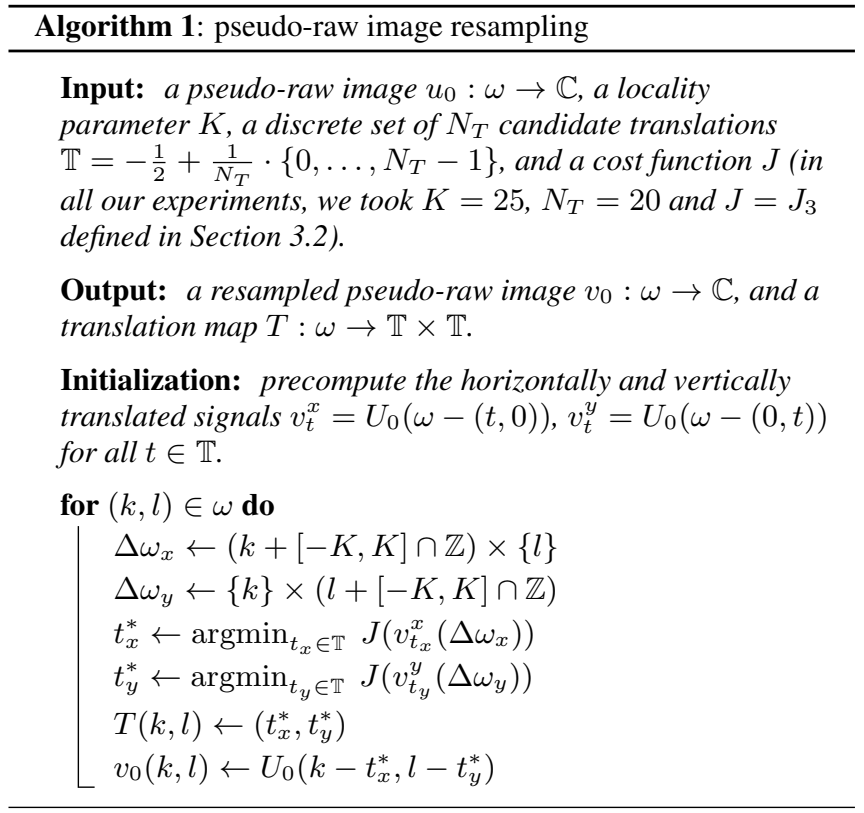




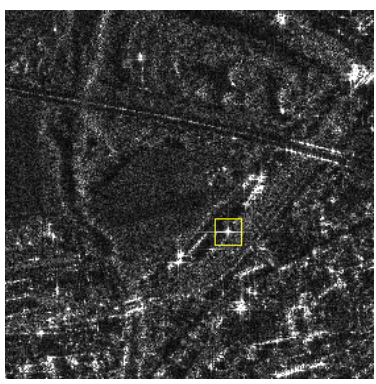

(a) pseudo-raw image $u_{0}$

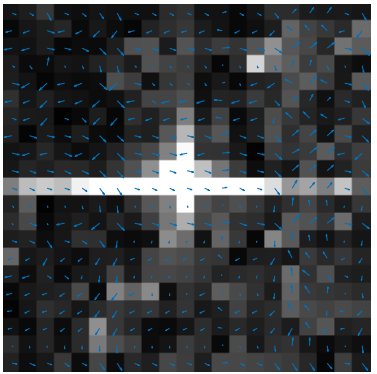

close-up view of (a)

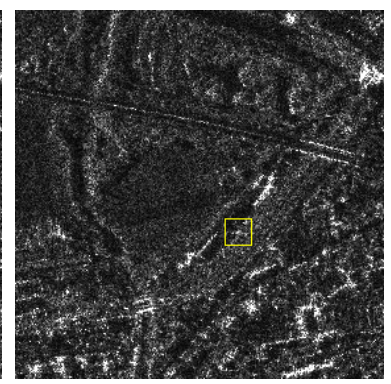

(b) resampled image $v_{0}$

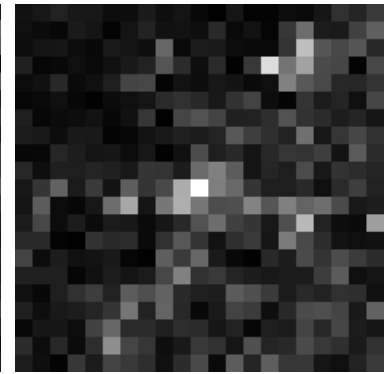

close-up view of (b)
Fig. 4. Resampling the pseudo-raw image. We display in (a) and (b) the modulus of the pseudo-raw image $u_{0}$ and the modulus of the resampled image $v_{0}$ computed using Algorithm 1. In the second row, we display some close-up views of the images (a) and (b). The blue arrows indicate the values of the computed translation fields $T$ at each pixel location. One can see that the resampled image $v_{0}$ exhibits a similar level of details than $u_{0}$ but is free of target induced cardinal sine phenomenon.

\subsection{Statistical properties of the resampled image}

We investigate the statistical properties of the resulting image and we show that, under a reasonable assumption, our sampling scheme produces a signal that is completely faithful to the underlying signal. We make the assumption that the scene is the superposition of a signal stemming from a bright target of amplitude $A$ and a fully-developed speckle. Under this model, the term $u_{0}^{*}(k)$ in (7) corresponds to the sampling of a band-limited Gaussian white second-order stationary process [6]. This means that the correlation between samples distant by an integer value is zero. The process being Gaussian, the zero correlation implies independence. A consequence of the bandlimited property of the SAR-signal is that the original discrete image allows one to recover the true values of $U_{0}(x)$ for real $x$ by means of Shannon interpolation of the available discrete signal. When examining the criteria $J_{1,2,3}$ proposed in Section 3.2, one can see that for the entire line the optimal value of $t_{x}$ is the same for all pixels, provided the computation window of the criterion is large enough. Thus, provided that our estimated $t_{x}$ equals to $\delta$ the final discrete result of our resampling will be, according to $(8), U_{0}^{*}(k+\delta)$ except at pixel $k=k_{0}$ (the target appears here) which are integer distant samples from the underlying fully-developed speckle and hence i.i.d Gaussian variables. We reduced the statistical accuracy of the resulting signal to the one of our estimator of $t_{x}$. One can show that this estimator is unbiased and has a variance depending on the ratio between the power of the $u_{0}^{*}$ process and $A$. In Fig. 6, we show how the output image $v_{0}$ both respects the theoretical Gaussian distribution of the samples and dramatically decreases the correlation between neighboring pixels, supporting our claim of statistical accuracy of the resampled image.

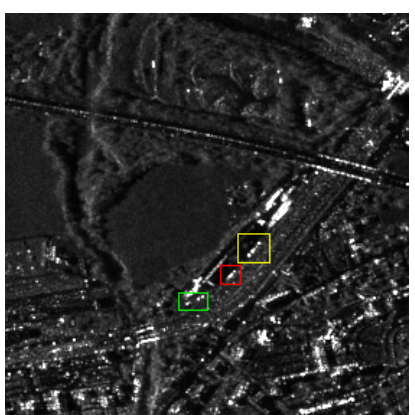

(a) apodized multi-look image

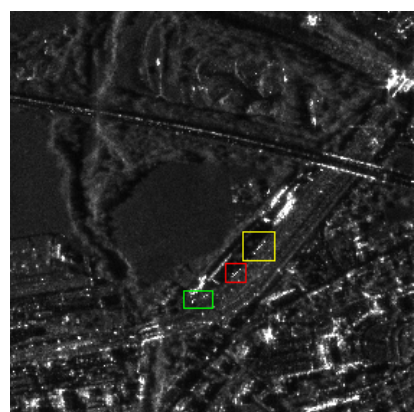

(b) resampled multi-look image
Fig. 5. Multi-Look comparison between apodized data and resampled pseudo-raw data. We display in (a) the multi-look realvalued image $u_{\omega}^{\mathrm{ML}}=\left(\frac{1}{26} \sum_{k=1}^{26}\left|u_{\omega}^{k}\right|^{2}\right)^{1 / 2}$ computed from a set of 26 views $\left\{u_{\omega}^{k}\right\}_{1 \leq k \leq 26}$, and in (b) the multi-look image computed from the corresponding set of resampled images $\left\{v_{0}^{k}\right\}_{1 \leq k \leq 26}$. The image (b) exhibits a better level of details and a better localization of the information than the image (a), as for instance in the areas delimited by the colored rectangles, where the strong targets can be more easily separated in (b) than in (a).
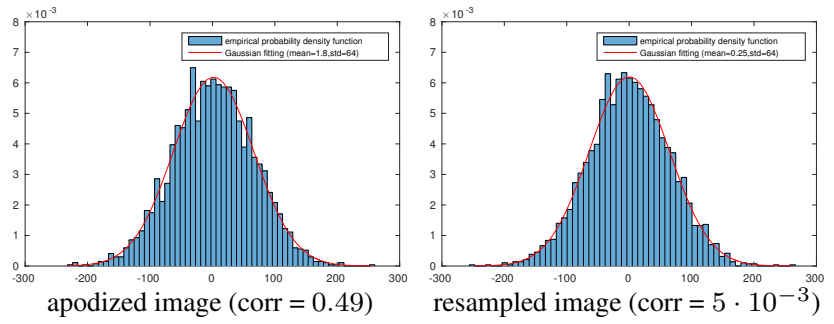

Fig. 6. First order statistics of a region with constant radiometry. We display here the empirical distribution of a $50 \times 60$ patch of the real part of the apodized image $u_{\omega}$ and the resampled image $v_{0}$ where the signal corresponds to pure speckle. In both cases, the empirical probability density function is very well approached by a Gaussian function. Pixels of $u_{\omega}$ are highly correlated (0.49) while those of $v_{0}$ exhibit a one hundred times smaller correlation (we computed $\operatorname{corr}(w)=\frac{1}{\|w\|^{2}} \sum_{k=0}^{K_{x}-2} \sum_{l=0}^{K_{y}-1} w(k+1, l) \cdot \bar{w}(k, l)$, where $w: I_{K_{x}} \times I_{K_{y}} \rightarrow \mathbb{C}$ denotes the considered patch).

\section{REFERENCES}

[1] D. Massonnet and J. C. Souyris, Imaging with Synthetic Aperture Radar, EPFL Press, 2008.

[2] Ch.-A. Deledalle, L. Denis, F. Tupin, A. Reigber, and M. Jäger, "NL-SAR: a unified Non-Local framework for resolutionpreserving (Pol)(In)SAR denoising," IEEE Trans. on Geoscience and Remote Sensing, vol. 53, no. 4, 2015.

[3] R. Abergel and L. Moisan, "The Shannon Total Variation," preprint MAP5, 2016.

[4] J. Li, "Implementation of the RELAX algorithm," Correspondence in IEEE Trans. on Aerospace and Electronic Systems, vol. 24, no. 2, 1998.

[5] J. Tsao and B. Steinberg, "Reduction of Sidelobe and Speckle Artifacts in Microwave Imaging: the CLEAN technique," IEEE Trans. on Antennas and Propagation, vol. 36, no. 4, 1988.

[6] C. Oliver and S. Quegan, Understanding Synthetic Aperture Radar Images, SciTech Publishing, 2004. 